\title{
Ensuring Trust in One Time Exchanges: Solving the QoS Problem
}

\author{
Bernardo A. Huberman, Fang Wu, and Li Zhang \\ \{bernardo.huberman, fang.wu, l.zhang\}@hp.com \\ HP Labs, Palo Alto, CA 94304
}

August 7, 2018

\begin{abstract}
We describe a pricing structure for the provision of IT services that ensures trust without requiring repeated interactions between service providers and users. It does so by offering a pricing structure that elicits truthful reporting of QoS by providers while making them profitable. This mechanism also induces truth-telling on the part of users reserving the service.
\end{abstract}




\section{Introduction}

A most important enabler of interactions over the Internet is the notion of trust, for it allows access to services, information, and customers without having to resort to validating procedures that can increase the complexity of an exchange so as to make it impractical. That is why reputations play an important role in deciding the level of trust in commercial exchanges, setting the value of particular brands, and in deciding whom to consult for professional advice.

A problem with reputations is that the time scales for their buildup or decay can be very long compared to the typical times involved in exchanges between a provider and a user [4, 9. This is not a problem if enough transactions have taken place so that a reputation or brand name can be established and made known to all parties concerned. In its place a number of trust management mechanisms for online environments have been proposed, ranging from the familiar reputation methods used by eBay, to the creation of trust records and model based compliance tools for the assurance of utility computing customers [1, 5].

In all these cases, either history or repeated interactions with a customer or system are necessary in order to establish a level of trust. But in many cases, especially those involving one time exchanges or new providers of services, the repeated interactions necessary to establish a reputation are not feasible. Indeed, there are many situations in online environments where no reputation is available while needing assurance that a particular quality of service (QoS) will be provided within a single exchange. An example could be access to a utility data center that promises a given QoS level, but the user cannot ascertain whether or not that level of service will be provided.

In this paper, we consider the most basic model for quality of service. In our model, a quality of service contract describes the likelihood that the service provider delivers the promised service. We have designed a mechanism that forces the provider to reveal his true assessment of the probability that he will be delivering a given service in a single interaction with a user/customer. We also solved the complementary truth telling reservation problem of obtaining from the user his assessment of the true probability that a given level of resources will be required at the time of their delivery. In both cases, our mechanisms use a contingent contract to elicit true revelation of both QoS and likelihood of use through a pricing structure that forces the parties to make accurate assessments of their ability to do what they commit to.

We also show that the combination of the two mechanisms, i.e. QoS and truth-telling reservations, provides an approach to solve the overbooking problem in reservation. The overbooking problem is when offering a service, the provider promises delivering more than he is actually able to do. The provider's incentive is based on the belief that some users may not actually use the service due to uncertainty about their own needs. However, overbooking can cause the provider to lose significantly if the user's needs exceeds the provider's capacity. In our case, by using truth telling reservations, the service provider can obtain better estimates of the number of users who actually need the service. We can 
then couple those estimates with a quality of service contract to deal with the overbooking problem.

Related work. There has been extensive work on the study of reputation as a basis for trust [1, 4, 5, 8, 9, Some recent work [7, 6] also describes mechanisms that let the users report their past experience truthfully so as to allow a more accurate buildup of reputations. As mentioned above, it requires a long time to establish reputations, and we are primarily interested in interactions for which there is not sufficient past history. There are also related papers in [2, 3] on trading mechanisms for trust revelation. They show that one can enforce truthful trust revelation through the amount of goods to be traded. In that work the setting is different from the problem we consider for there is no flexibility on the amount of goods the buyer purchases. Instead, we use contingent contracts to let the service provider reveal the quality of service.

\section{The Quality of Service (QoS) Problem}

\subsection{The problem}

Consider the following one-time exchange problem between two parties: a user and a service provider. The user is interested in buying a service from the provider. Because of physical constraints, the service provider cannot always provide the service to a satisfactory level, but can only do so with a probability $q \in[0,1]$, which we call the quality of service $(Q o S)$. Suppose the service provider knows the real $q$ but the user does not. We wish to design a mechanism that induces the service provider to report the true QoS to the user.

\subsection{The mechanism}

We describe a contingency mechanism:

1. The service provider tells the user his QoS $q^{\prime}$, which may or may not be the real QoS $q$.

2. The user pays the service provider a premium $g\left(q^{\prime}\right)$.

3. If the service provider fails to provide the service (to a satisfactory level), he pays the user a compensation $h\left(q^{\prime}\right)$.

Here $g, h:[0,1] \rightarrow \mathbb{R}^{+}$are two functions whose forms are to be determined.

For a risk-neutral user, he has the following form of expected utility:

$$
\mathbb{E} U_{1}=q v-g\left(q^{\prime}\right)+(1-q) h\left(q^{\prime}\right),
$$

where $v>0$ is his value of using the service, and $g\left(q^{\prime}\right)-(1-q) h\left(q^{\prime}\right)$ is his expected cost. Similarly, we assume that the service provider is risk-neutral, and his expected utility is given by

$$
\mathbb{E} U_{2}=g\left(q^{\prime}\right)-(1-q) h\left(q^{\prime}\right)-c,
$$


where $c \geq 0$ is his cost. Here we have assumed that $c$ is a fixed cost ex ante. That is, the service provider has to spend this cost whether or not he can successfully provide the service later. We further assume $c<v$ for there to be business.

Suppose furthermore that both parties are rational, so they maximize their expected utilities respectively. In particular, the service provider will report the $q^{\prime}$ that maximizes Eq. (2). Since we wish to induce him to report the true QoS, Eq. (2) should be maximized at $q^{\prime}=q$. This naturally leads to the following definition:

Definition 1. $(g, h)$ is called truth-telling on an interval $I \subseteq[0,1]$ if for any $q \in I$ and any $q^{\prime} \in[0,1]$ with $q^{\prime} \neq q$

$$
w(q) \equiv g(q)-(1-q) h(q)>g\left(q^{\prime}\right)-(1-q) h\left(q^{\prime}\right) .
$$

From this definition, the service provider will report the true QoS if it is in the truth-telling interval of $(g, h)$. If that happens, his expected income is $w(q)$. Clearly, he prefers using the mechanism to not selling any service when $w(q) \geq c$. On the other hand, knowing that the service provider will report the real $q$, the user prefers using the mechanism to staying idle when $w(q) \leq v$. We thus make the second definition:

Definition 2. $(g, h)$ is called incentive compatible on an interval $I \subseteq[0,1]$ if $c \leq w(q) \leq q v$ for all $v \in I$.

To summarize, if $(g, h)$ is truth-telling and incentive compatible on some interval $I \subseteq[0,1]$, then whenever $q \in I$, the user and the service provider both want to use the mechanism, and the service provider reports his real QoS. In the following, we will give examples of such pairs of $(g, h)$.

\subsection{Realizations}

In this section we give concrete realizations of the functional form $(g, h)$ that are both truth-telling and incentive compatible. Such pairs are not unique. We give two families of designs with different forms of compensation. The most intuitive choice is to require the compensation proportaional to the quality of service. Such linear compensation scheme is appropriate for non mission critical services.

Proposition 1 (Linear compensation). Suppose $k, c_{1}$ are positive numbers satisfying $c \leq c_{1} \leq v-k$. Let

$$
q_{0}=\frac{v-\sqrt{v^{2}-4 k c_{1}}}{2 k} .
$$

Then the choice

$$
g(q)=-k q^{2}+2 k q+c_{1}, \quad h(q)=2 k q
$$

is truth-telling and incentive compatible on $\left[q_{0}, 1\right]$. 
Proof. Clearly $g(q), h(q) \geq 0$ for all $q \in[0,1] .(g, h)$ is truth-telling because

$$
g\left(q^{\prime}\right)-(1-q) h\left(q^{\prime}\right)=-k\left(q^{\prime}-q\right)^{2}+k q^{2}+c_{1}
$$

is maximized at $q^{\prime}=q$ with the value $w(q)=k q^{2}+c_{1}$. The incentive compatibility part is just some basic algebra. Figure 1a) plots the function $g, h$ for a particular set of parameters.

The parameter $k$ determines the curvature of the function $g$ : the larger $k$, the more curved is $g$.

The linear compensation scheme is not appropriate for the services which require high reliability. In the areas such as in telecommunications and in financial IT services, the quality of service is often represented by the "number of nines" that measures how close it is to 1 . For example "five nines" is equivalent to $q=0.99999$. For these services it is more appropriate to set the compensation proportional to $-\log (1-q)$. This leads to the following design.

Proposition 2 (Logarithmic compensation). Suppose $k, c_{1}$ are positive numbers satisfying $c \leq c_{1} \leq v-k$. Let $q_{0}=\left(c_{1}+k\right) / v$. Then the choice

$$
g(q)=k q+c_{1}, \quad h(q)=-k \log (1-q)
$$

is truth-telling and incentive compatible on $\left[q_{0}, 1\right)$.

Proof. Under our assumptions $g(q), h(q) \geq 0$ for $q \in[0,1)$. Consider the function

$$
G\left(q^{\prime}\right)=g\left(q^{\prime}\right)-(1-q) h\left(q^{\prime}\right)=k q^{\prime}+c_{1}+k(1-q) \log \left(1-q^{\prime}\right) .
$$

First order condition

$$
G^{\prime}\left(q^{\prime}\right)=k \frac{q-q^{\prime}}{1-q^{\prime}}=0
$$

is satisfied when $q^{\prime}=q$. Since

$$
G^{\prime \prime}\left(q^{\prime}\right)=-k \frac{1-q}{\left(1-q^{\prime}\right)^{2}}<0,
$$

$G\left(q^{\prime}\right)$ is maximized at $q^{\prime}=q$ with the maximum value

$$
w(q)=k(1-q) \log (1-q)+k q+c_{1} .
$$

Thus $(g, h)$ is truth-telling on $[0,1)$.

Because

$$
w^{\prime}(q)=-k \log (1-q) \geq 0,
$$

$w(q)$ is nondecreasing on $[0,1)$. By our assumptions

$$
\begin{aligned}
w(0) & =c_{1} \geq c, \\
w(1-) & =c_{1}+k=q_{0} v .
\end{aligned}
$$

Thus $(g, h)$ is incentive compatible on $\left[q_{0}, 1\right)$. 


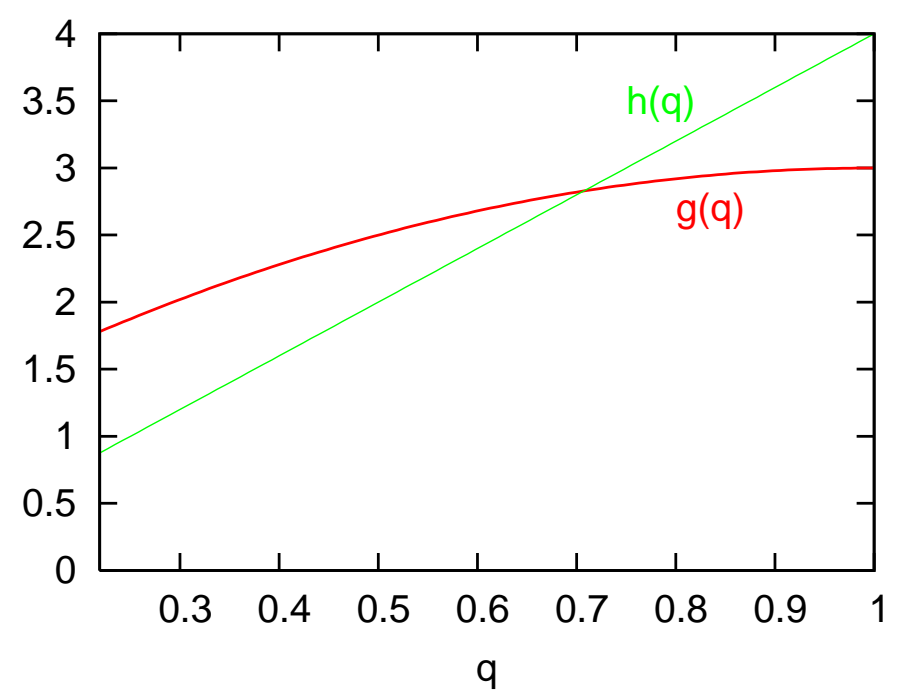

(a)

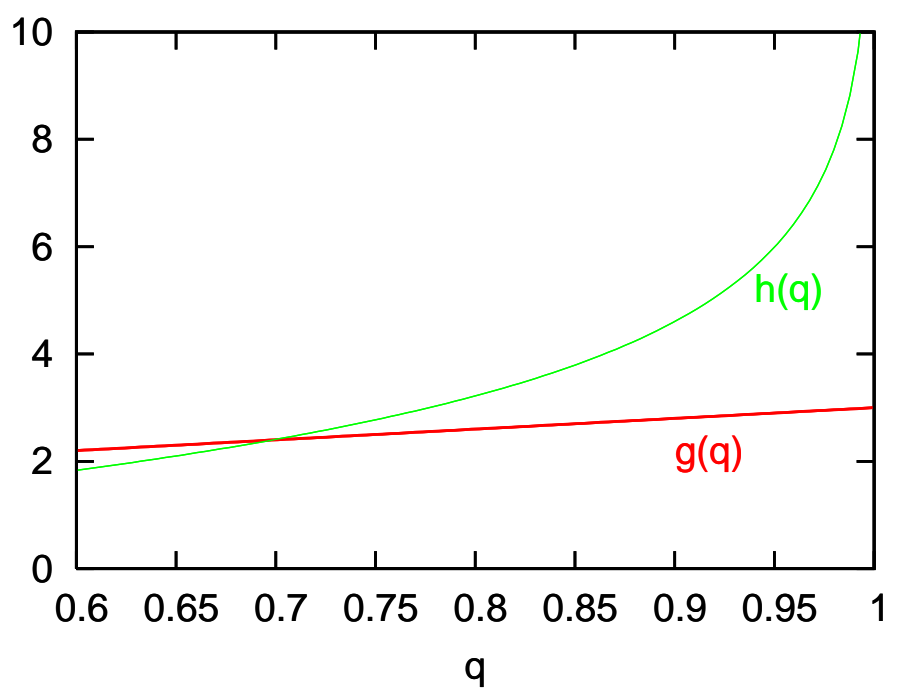

(b)

Figure 1: Plot of the premium and compensation functions for (a) linear compensation scheme; and (b) logarithmic compensation scheme. The parameters for both figures are $c=1, v=5, k=2, c_{1}=1$. The incentive compatible interval is $[0.219,1]$ for $(\mathrm{a})$ and $[0.6,1]$ for $(\mathrm{b})$. The curve $g(q)$ is the premium and $h(q)$ is the compensation. 
Remark. In both examples, the pairs $(g, h)$ is truth telling in the full interval $[0,1]$. It is the incentive compatibility requirement that restricts the applicable interval. Therefore, once the service provider decides to participate, we expect him to be truth-telling. In both examples, $q_{0} \rightarrow 0$ as $v \rightarrow \infty$. In words, the more valuable the service, the larger is the incentive compatible interval.

\section{The reservation problem}

\subsection{The problem}

In the last section we described QoS as the probability that the service provider can successfully provide the service. The probability $q$ thus captures the random nature of supply. We now introduce a second probability $p$ to describe the randomness of demand. For simplicity, assume that there are two periods: 1 and 2. With probability $p$ the user will need one unit of service in period 2 , and he knows this probability of needing it in period 1 . The service provider wishes to know the real $p$ in period 1 so that he can set up the correct quota of service beforehand at a relatively low cost. In what follows we describe a contingent contract that incentivizes the user to report his probability of usage truthfully.

\subsection{The mechanism}

In what follows we will use $p$ and $q$ to denote real probabilities, and use $p^{\prime}$ and $q^{\prime}$ to denote proposed probabilities.

1. (Period 1) The service provider tells the user his QoS $q^{\prime}$, which may or may not be his real QoS $q$.

2. (Period 1) The user tells the service provider a probability $p^{\prime}$ that he will need the service in period 2, which may or may not be equal to $p$.

3. (Period 1) The user pays the service provider a premium $g\left(p^{\prime}, q^{\prime}\right)$.

4. (Period 1) The service provider prepares $p^{\prime}$ unit of service for the user.

5. (Period 2) If the service provider cannot provide the service (which happens with probability $1-q$ ), he pays the user a compensation $h\left(p^{\prime}, q^{\prime}\right)$. Otherwise, the user can get the service at the price $f\left(p^{\prime}\right)$ if he needs it.

Using the mechanism, the user's expected cost is

$$
\mathbb{E} C\left(p^{\prime}, q^{\prime}\right)=g\left(p^{\prime}, q^{\prime}\right)+p q f\left(p^{\prime}\right)-(1-q) h\left(p^{\prime}, q^{\prime}\right) .
$$

In period 2 , the user gets value $v$ if he needs the service and can obtain it. Hence his value in period 1 can be described by a random variable

$$
V= \begin{cases}v & \text { with probability } p q, \\ 0 & \text { with probability } 1-p q .\end{cases}
$$


So his expected utility in period 1 is

$$
\mathbb{E} U_{1}=\mathbb{E}[V-C]=p q v-\mathbb{E} C .
$$

The service provider collects $\mathbb{E} C$ from each user in expectation and his cost is $c p$. Thus his expected utility is

$$
\mathbb{E} U_{2}=\mathbb{E} C-c p .
$$

If both parties are rational and risk-neutral, then the service provider maximizes $\mathbb{E} C\left(p^{\prime}, q^{\prime}\right)$ for $q^{\prime}$ while the user minimizes $\mathbb{E} C\left(p^{\prime}, q^{\prime}\right)$ for $p^{\prime}$. So they are playing a constant sum game.

Similar to the pure QoS problem, we make two definitions:

Definition 3. $(g, f, h)$ is called truth-telling on a region $I \subseteq[0,1]^{2}$ if for any $(p, q) \in I$ and any $\left(p^{\prime}, q^{\prime}\right) \in[0,1]^{2}$ with $\left(p^{\prime}, q^{\prime}\right) \neq(p, q)$

$$
\begin{aligned}
w(p, q) & \equiv g(p, q)+p q f(p, q)-(1-q) h(p, q) \\
& >g\left(p^{\prime}, q^{\prime}\right)+p q f\left(p^{\prime}, q^{\prime}\right)-(1-q) h\left(p^{\prime}, q^{\prime}\right) .
\end{aligned}
$$

Definition 4. $(g, f, h)$ is called incentive compatible on a region $I \subseteq[0,1]^{2}$ if $c p \leq w(p, q) \leq p q v$ for all $(p, q) \in I$.

According to the above definition, if $(g, f, h)$ is truth-telling and incentive compatible on some region $I \subseteq[0,1]^{2}$, then whenever $(p, q) \in I$, the user and the service provider both want to use the mechanism. The user reports his true probability of usage, and the service provider reports his real QoS. We will show such triplet $(g, f, h)$ does exist.

\subsection{Realization}

Following the linear compensation scheme, we can design the following functions which are truth-telling and incentive compatible.

Proposition 3. Consider the following choice of $(g, f, h)$ :

$$
\begin{aligned}
g\left(p^{\prime}, q^{\prime}\right) & =k_{1} p^{2}-k_{2} q^{2}+2 k_{2} q^{\prime}+c_{1}, \\
f\left(p^{\prime}\right) & =-2 k_{1} p^{\prime}+c_{2}, \\
h\left(p^{\prime}, q^{\prime}\right) & =k_{1} p^{\prime 2}+2 k_{2} q^{\prime}+c_{3},
\end{aligned}
$$

where $k_{1}, k_{2}, c_{1}, c_{2}, c_{3}$ are positive numbers satisfying $c_{2} \geq 2 k_{1}, c_{1}-c_{3} \geq c$ and $c<c_{1}+c_{2}-k_{1}+k_{2}<v$. Then there exist $p_{0}<1$ and $q_{0}<1$ such that $(g, f, h)$ is truth-telling and incentive compatible on $\left[p_{0}, 1\right] \times\left[q_{0}, 1\right]$. Furthermore $p_{0}, q_{0} \rightarrow 0$ as $v \rightarrow \infty$.

Proof. It can be calculated that for our choice of $(g, f, h)$

$$
\mathbb{E} C\left(p^{\prime}, q^{\prime}\right)=k_{1} q\left(p^{\prime}-p\right)^{2}-k_{2}\left(q^{\prime}-q\right)^{2}-k_{1} p^{2} q+k_{2} q^{2}+c_{3} q+c_{2} p q+c_{1}-c_{3},
$$


so $\left(p^{\prime}, q^{\prime}\right)=(p, q)$ is a saddle point of $\mathbb{E} C\left(p^{\prime}, q^{\prime}\right)$. Thus $(g, f, h)$ is truth-telling on $[0,1]^{2}$.

From Eq. (24) we see that

$$
w(p, q)=-k_{1} p^{2} q+k_{2} q^{2}+c_{3} q+c_{2} p q+c_{1}-c_{3} .
$$

By assumption $c p<w(p, q)<v p q$ at $p=q=1$. By continuity, there exists a region $\left[p_{0}, 1\right] \times\left[q_{0}, 1\right]$ on which $c p<w(p, q)<v p q$.

Next we show that for any $p_{0}, q_{0}>0$ there exists $v$ large enough such that $(g, f, h)$ is incentive compatible on $I=\left[p_{0}, 1\right] \times\left[q_{0}, 1\right]$. Since $I$ is compact, the continuous function $w(p, q)$ achieves its maximum value $w_{M}$ on $I$. Pick $v$ so large that $v p_{0} q_{0} \geq w_{M}$, and we have $w(p, q) \leq w_{M} \leq v p_{0} q_{0} \leq v p q$ for $(p, q) \in I$. For the other direction, note that

$$
\frac{\partial w}{\partial q}=c_{2} p-k_{1} p^{2}+2 k_{2} q+c_{3}>0,
$$

so $w$ is increasing in $q$. Therefore for $(p, q) \in I$ we have

$$
w(p, q) \geq-k_{1} p^{2} q_{0}+k_{2} q_{0}^{2}+c_{3} q_{0}+c_{2} p q_{0}+c_{1}-c_{3}>c_{1}-c_{3} \geq c p .
$$

Hence $(g, f, h)$ is incentive compatible on $I$.

Remark. We note that in the above proof, it is relatively easier to argue the truth-telling property. However, it is harder to prove the incentive compatibility and to calculate the analytical form of the interval in which the scheme is incentive compatible. It is possible to design schemes by using the logarithmic compensation function. It is considerably more challenging for proving the incentive compatibility.

\section{Application to finite resource reservation}

Besides the obvious advantages that our mechanism brings to the problem of determining QoS in single exchanges and truth-telling in reservations, there are other scenarios which can also profit from it. These scenarios are characterized by the fact that resources are finite and thus they can lead to severe overbooking problems. For example, consider the case where there are $m$ available units of resource (plane seats, conference rooms, etc.) and $n$ users. Each user may need to consume one unit in period 2. If $n \leq m$ then our reservation mechanism for infinite resource works without problems. If $n>m$, however, the service provider can no longer guarantee the delivery of one unit in period 2 , even if the user reserved in period 1.

This is known as the overbooking problem in a reservation system. One existing method, used for example in the airline industry, for dealing with the overbooking problem is by holding an auction at period 2 until enough users surrender their reservations for monetary compensation. This process is often 
expensive to the service provider, and can be gamed by some users willing to benefit from such compensation. Our mechanism can solve this in the following fashion.

Suppose $n$ users arrive and make reservations one after another. In period 2 when they claim their needs, they are satisfied one by one in the order of their arrivals, until there are no more available resources left. The first $m$ users for sure can be satisfied, so in period 1 the coordinator simply sells them $m$ plain truth-telling options with $q=1$ as in the infinite resource problem, and the users truthfully report their probabilities of needing one unit of resource in period 2 , which we call $p_{1}, \ldots, p_{m}$.

Now consider the $(m+1)$ 'th user. The first $m$ probabilities $p_{1}, \ldots, p_{m}$ known by the service provider can help him to estimate the $\operatorname{QoS} q_{m+1}$ for the $(m+1)^{\text {'th }}$ user. For example, under the assumption that the users' usage probabilities are independent, with probability $p_{1} \cdots p_{m}$ the first $m$ users will each need one unit of resource in period 2. If that happens, user $m+1$ cannot be satisfied. Therefore, the probability $q_{m+1}=1-p_{1} \cdots p_{m}$ can be regarded as the QoS for the $(m+1)$ 'th user. Thus, the coordinator sells the $(m+1)$ 'th user an option with QoS $q_{m+1}$, as specified in Section [3 and the $(m+1)$ 'th user reports his true probability of using one unit of resource in period 2, denoted by $p_{m+1}$. Once the coordinator knows $p_{m+1}$, he can calculate the quality of service for the $(m+2)$ 'th user, and can sell the $(m+2)$ 'th user an option with QoS $q_{m+2}$ and so on. If everyone is risk-neutral and rational, then all the probabilities $p$ and $q$ will be truthful.

The effectiveness of the procedure just described depends on the accuracy of the estimation of $q_{i}$ from $p_{1}, \ldots, p_{i-1}$, a problem similar to time-series predictions. If the estimation process is not designed appropriately, the error may accumulate and propagate to the very last step, i.e. the service provider will systematically err in estimating QoS for all subsequent users. In addition to usage probabilities, the service provider may also take into account the historical data.

Nevertheless in all other cases, including those where the constraint of finite resources is not severe, we believe that our contingent contracts can provide an efficient procedure for delivering services without resorting to either long histories of auction systems that can be gamed.

\section{Conclusion}

In this paper we presented a mechanism that forces a service provider to reveal his true assessment of the probability that he will be delivering a given service in a single interaction with a user/customer. We also solved the complementary truth telling reservation problem of obtaining from the user his assessment of the true probability that a given level of resources will be required at the time of their delivery. In both cases the mechanism elicits true revelation of both QoS and likelihood of use through a pricing structure that forces the parties to make accurate assessments of their ability to do what they commit to. 
Throughout, we considered the most basic model for quality of service, one in which the service contract describes the likelihood that the service provider delivers the promised service. We also showed that the combination of the two mechanisms, i.e. QoS and truth-telling reservations, provides an elegant approach to the overbooking problem in reservation.

There are ongoing experiments with human subjects to verify the effectiveness of these mechanisms. The initial results are encouraging, and the detailed experimental results will soon be reported. We also plan to apply the scheme to practical IT service reservations and to design concrete schemes that are appropriate for different scenarios.

\section{References}

[1] A. Baldwin, Y. Beres, D. Plaquin, and S. Shiu. Trust record: High-level assurance and compliance. In Proceedings of the 3rd International Conference on Trust Management, May 2005.

[2] S. Brainov. An incentive compatible trading mechanism for trust revelation. In Proceeding of the IJCAI'01 Workshop Economic Agents, Models and Mechanisms, pages 62-70, 2001.

[3] S. Braynov and T. Sandholm. Contracting with uncertain level of trust. Computational Intelligence, 18(4):501-514, November 2002.

[4] B. A. Huberman and F. Wu. The dynamics of reputation. In Proceedings of the International Conference on Computing in Economics and Finance, 2003.

[5] A. Josang, C. Keser, and T. Dimitrakos. Can we manage trust? In Proceedings of the 3rd International Conference on Trust Management, May 2005.

[6] R. Jurca and B. Faltings. An incentive compatible reputation mechanism. In Proceedings of the IEEE Conference on E-Commerce, Newport Beach, CA, USA, June 24-27 2003.

[7] R. Jurca and B. Faltings. Enforcing truthful strategies in incentive compatible reputation mechanisms. In Proceedings of the Workshop on Internet and Network Economics, Hong Kong, China, 2005.

[8] N. Miller, P. Resnick, and R. Zeckhauser. Eliciting honest feedback: The peer prediction method. To appear in Management Science.

[9] P. Resnick, R. Zeckhauser, R. Friedman, and K. Kuwabara. Reputation systems. Communications of the ACM, 43:45-48, 2000. 